\title{
Visual search and eye movements in novel and familiar contexts
}

\author{
Kyle McDermott ${ }^{\mathrm{a}}$, Jeffrey B. Mulligan ${ }^{\mathrm{b}}$, George Bebis ${ }^{\mathrm{c}}$, Michael A. Webster*a \\ ${ }^{a}$ Department of Psychology, University of Nevada, Reno, Reno NV 89557 \\ ${ }^{b}$ NASA Ames Research Center, Moffett Field, CA 94035 \\ ${ }^{\mathrm{c}}$ Department of Computer Science and Engineering, University of Nevada, Reno, Reno NV 89557
}

\begin{abstract}
Adapting to the visual characteristics of a specific environment may facilitate detecting novel stimuli within that environment. We monitored eye movements while subjects searched for a color target on familiar or unfamiliar color backgrounds, in order to test for these performance changes and to explore whether they reflect changes in salience from adaptation vs. changes in search strategies or perceptual learning. The target was an ellipse of variable color presented at a random location on a dense background of ellipses. In one condition, the colors of the background varied along either the LvsM or SvsLM cardinal axes. Observers adapted by viewing a rapid succession of backgrounds drawn from one color axis, and then searched for a target on a background from the same or different color axis. Searches were monitored with a Cambridge Research Systems Video Eyetracker. Targets were located more quickly on the background axis that observers were pre-exposed to, confirming that this exposure can improve search efficiency for stimuli that differ from the background. However, eye movement patterns (e.g. fixation durations and saccade magnitudes) did not clearly differ across the two backgrounds, suggesting that how the novel and familiar backgrounds were sampled remained similar. In a second condition, we compared search on a nonselective color background drawn from a circle of hues at fixed contrast. Prior exposure to this background did not facilitate search compared to an achromatic adapting field, suggesting that subjects were not simply learning the specific colors defining the background distributions. Instead, results for both conditions are consistent with a selective adaptation effect that enhances the salience of novel stimuli by partially discounting the background.
\end{abstract}

Keywords: vision, adaptation, eye movements, visual search

\section{INTRODUCTION}

A very large body of literature is devoted to the question of what aspects of a scene can capture attention ${ }^{1}$. These aspects include goals of the observer (e.g. whether they are searching for a specific target ${ }^{2}$ ) and properties of the stimulus ${ }^{3}$. For instance, elements of a scene that are very different from their background tend to be highly salient and thus "pop out" of the display ${ }^{4}$. This pop-out typically occurs for stimuli that are very different from their background in a simple feature dimension such as color or shape. On the other hand, stimuli that are distinct in more complex ways can often go undetected. Classic examples of these are stimuli defined only by a unique combination or conjunction of features ${ }^{5}$. More recent work has shown that observers often fail to notice large physical changes introduced into scenes ${ }^{6}$ Such studies of "change blindness" suggest that observers normally encode only the "gist" "or immediate task relevance ${ }^{8}$ of the stimulus, while remaining unaware of much of the specific detail. In terms of the stimulus, one way to conceptualize such effects is that the visual system may represent only the statistical structure of scenes, and thus scene changes that do not alter these statistics are not salient ${ }^{9}$. Conversely, statistical outliers represent novel and thus unexpected image properties or suspicious objects. It makes sense to allocate attention to these, because they represent aspects of the image which do not fit the observer's expectations about the environment, and which thus may be more informative ${ }^{10}$.

In this study we examined how processes of visual adaptation might influence the detection of statistical outliers, or novelty. Obviously, the ability to identify a novel property rests on the knowledge that observers have about the environment. Incorporating knowledge about the world is thought to be one of the most important principles governing the evolutionary design of the visual system ${ }^{11}$. However, the problem arises that the world itself is not static, and instead can vary considerably across different environments, or even in the same environment over time ${ }^{12}$. Thus it is not possible

*for correspondence contact MAW at mwebster@unr.nevada.edu 
in principle to build a static visual system that is optimized for all of the viewing situations that the observer may routinely encounter. A second fundamental design principle in sensory systems is thus that they must be adaptable, in order to recalibrate their response properties in order to match properties of the current environment. Barlow ${ }^{13}$ proposed that a major function of such adjustments is that they may aid the visual detection of new stimulus properties by discounting the recurring characteristics of the environment. That is, adaptation may allow the observer to build a prediction about the environment, and thus draw attention to the errors in this prediction.

Few studies have directly tested this putative role of adaptation. However, in a previous study, Webster, Raker, and Malkoc ${ }^{14}$ examined whether adaptation increased the salience of novel stimuli by using a visual search task, in which subjects had to find a colored target presented at random locations on a cluttered color background. The background was composed of a dense array of overlapping ellipses with colors selected from a pre-defined distribution, while the target was a superimposed circle with variable color. The experiment thus simulated a "foraging" task in which observers searched for a "fruit" among a variegated background of foliage. In this task, search times systematically decreased as the difference between the target color and the background colors increased. However, observers were consistently faster at finding a given target color if they were first adapted to the set of background colors (by viewing random samples of the background for a period of time before searching), and were consistently slower if they were instead adapted to a different (orthogonal) set of colors. These results thus supported Barlow's suggestion that adaptation might make novel stimulus properties more conspicuous by selectively reducing sensitivity to the properties of the ambient background. However, these measures of search times alone cannot rule out other explanations for the performance improvements, and thus leave open the nature of the "adaptation" effects in such tasks. Specifically, they did not distinguish between changes in how visible the targets were vs. how subjects scanned the backgrounds for them, and did not distinguish between actual sensory adaptation to the background vs. learning about the background.

In the present study, we repeated the visual search task used by Webster et al. ${ }^{14}$, but measured the pattern of eye movements during the search. Eye movements provide a rich source of information about how individuals acquire information and carry out visual tasks ${ }^{15,16}$, and have been analyzed previously to characterize the dynamics of visual $\operatorname{search}^{17-20}$. In our case we used them to explore how visual search is influenced by prior adaptation to the backgrounds. In particular, we asked whether an adaptation effect on the search times could be accounted for by changes in how observers might sample familiar vs. unfamiliar backgrounds, or by a change in the effective contrast of the backgrounds. This was assessed by comparing the statistics of the fixations for adapted vs. unadapted background conditions. Our results confirm that prior adaptation to the background on which observers are searching facilitates the detection of novel target colors, but reveal little difference between the search strategies on the familiar and unfamiliar backgrounds. That is, observers scanned the two backgrounds in the same way, but were faster at finding the target on the adapted background, consistent with a change in sensitivity rather than strategy. In a second condition, we further evaluated the nature of the adaptation by measuring search times for targets on backgrounds drawn from all color directions. Adaptation to these nonselective backgrounds should reduce sensitivity to both the background and the target, and thus would not be predicted to facilitate detection of the target. On the other hand, if subjects learn which colors are in the backgrounds, then they might become better at spotting a novel color. Our results show that prior exposure to a nonselective color distribution does not facilitate search, and thus are again consistent with the predictions for contrast adaptation. Together these findings help characterize how visual salience and visual exploration might differ in novel or familiar contexts, and suggest that much of this difference might be predictable from visual adaptation.

\section{METHODS}

Apparatus. Stimuli were shown on a SONY 20SE monitor controlled by a Cambridge Research Systems VSG graphics card. Subjects viewed the screen from a distance of $57 \mathrm{~cm}$ with their heads resting on the chin rest of a Cambridge Research Systems Video Eyetracker. The right eye was tracked during each search.

Stimuli. Search was measured for a unique color target presented at a random location on a selected background of colors (Fig 1). The background subtended 41 by $31 \mathrm{deg}$ and was composed of a dense set of ellipses. Each ellipse had a 


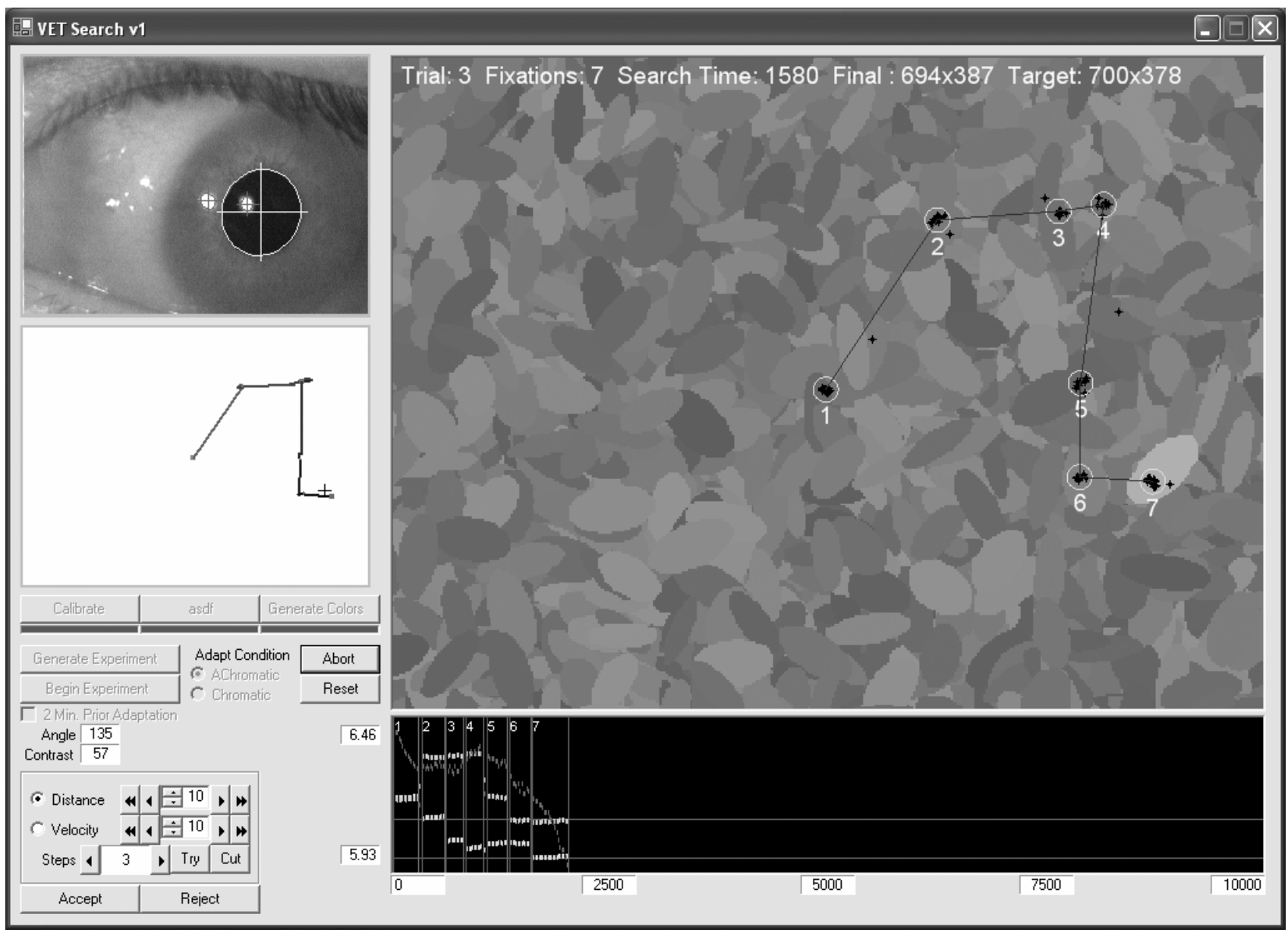

Figure 1. Analysis screen showing the search background and measured fixations. In this example the target (highlighted ellipse) was located on the 7 th fixation.

randomly chosen orientation and a size selected at random from between 0.2-0.4 deg for its minor axis and 0.6-0.8 deg for the major axis. A total of 10,000 ellipses were generated in order to completely fill the background, with most ellipses partially or wholly occluded. In order to rapidly resample backgrounds during the experiment, a single background was stored in video memory that was 8.7 times as large as the display. Different samples from the background could then be shown by zooming a random area within the stored background.

The color of each ellipse was also chosen at random by selecting values along one of two "cardinal" axes in color space: an LvsM axis that varies opposing signals in the $\mathrm{L}$ and $\mathrm{M}$ cones, or an SvsLM axis that varies signals in the $\mathrm{S}$ cones opposed by the sum of signals from the $\mathrm{L}$ and $\mathrm{M}$ cones. Backgrounds defined by the LvsM axis appeared to vary between different saturations of red and blue-green, while the SvsLM background varied between purple and yellowgreen. Early stages of postreceptoral color coding are thought to be tuned primarily to these two orthogonal color directions $^{21}$, and color contrast adaptation is known to be strongly selective for these axes ${ }^{22,23}$. That is, adaptation to the LvsM axis produces much larger changes in sensitivity and appearance to LvsM contrasts than to SvsLM contrasts, and vice versa. Contrasts along the two axes spanned a range of \pm 80 units in a color space that was scaled to roughly equate color differences along the two directions. Units in this space are related to the r,b coordinates of the MacLeodBoynton $^{24}$ chromaticity diagram by the following equations:

$$
\begin{aligned}
\operatorname{LvsM} & =(\mathrm{r}-0.6568) * 1955 \\
\text { SvsLM } & =(\mathrm{b}-0.01825) * 5533
\end{aligned}
$$



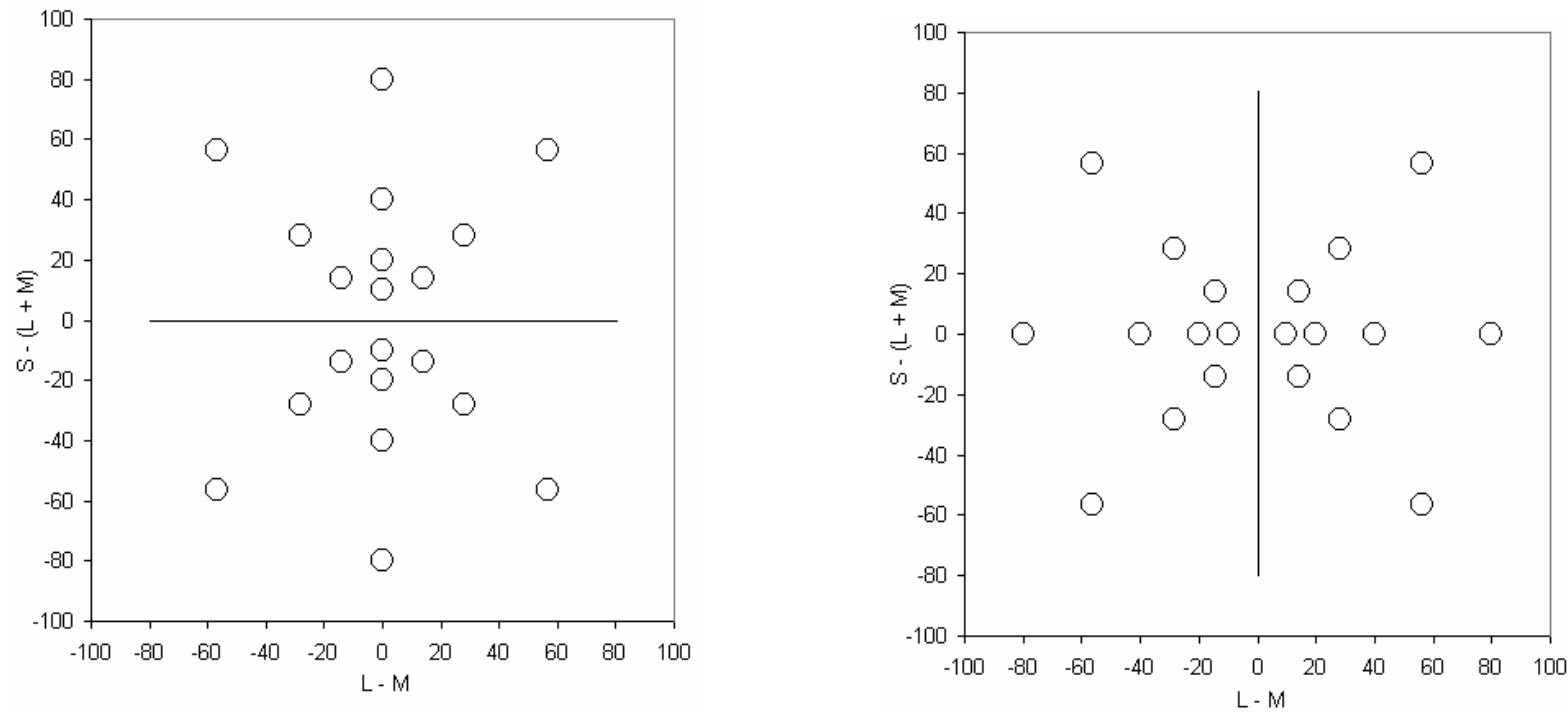

Figure 2. Color coordinates of the targets and backgrounds. Background colors varied along either the LvsM axis (left panel) or the SvsLM axis (right panel).

Where $r, b=(0.6568,0.01825)$ are the chromaticity coordinates of the white point (equivalent to illuminant $C)$ which remained the average chromaticity for all conditions. Finally, each background ellipse also varied randomly in luminance over a range of $\pm 40 \%$ of the mean luminance, which was maintained at $25 \mathrm{~cd} / \mathrm{m}^{2}$.

Target stimuli varied in color along both cardinal and intermediate axes and over a range of contrasts from 10 to 80 (Fig 2). Unlike the previous study of Webster et al. ${ }^{14}$, the target was also an ellipse rather than a circle, and thus there were no reliable shape cues for distinguishing the target. This had the advantage that search times in all cases depended on the color characteristics of the pattern, but limited the target colors to be stimuli that were not contained within the background axis.

Procedure. Each run began with a 2 min period of adaptation to backgrounds drawn from a single color axis. During the adaptation, the background was resampled every $750 \mathrm{~ms}$, so that observers were exposed and thus adapted to the global statistics of the background rather than an individual and fixed image. A test trial was next initiated by displaying a background shown with a small central cross, which subjects were instructed to fixate. This pretest field was shown for $1 \pm 0.5 \mathrm{sec}$ and was also marked by a tone. The target then appeared on a new background, and was placed at a random location between 5.1 and $15.3 \mathrm{deg}$ from the center of the display. The stimulus remained on the screen while subjects searched for the target, during which eye movements were recorded, and the trial was terminated when the target was located (as determined by the measured fixations). Some trials were also terminated after $10 \mathrm{sec}$ if the target was not located. Subjects readapted to the adapting background for $6 \mathrm{sec}$ before the next trial, and the sequence continued until each target color had been shown on each of the two possible test backgrounds (i.e. the adapting background or the orthogonal background) twice. On each run the order of targets and backgrounds was randomized. Subjects were tested after adapting to either of the two backgrounds shown in counterphased order, and completed 10 runs so that at least 10 searches were completed for each target on each background condition. The subjects included two of the authors (KM and MW) and 6 additional observers who were naïve with respect to the specific aims of the study.

Eye movement analysis. We used eye movements to track how the subjects scanned the backgrounds to locate the target and to determine when the target was detected. A typical example of these eye movements is illustrated by the scan path plotted in Figure 1, which was taken from an actual trial. Successful detection was defined by maintaining fixation 

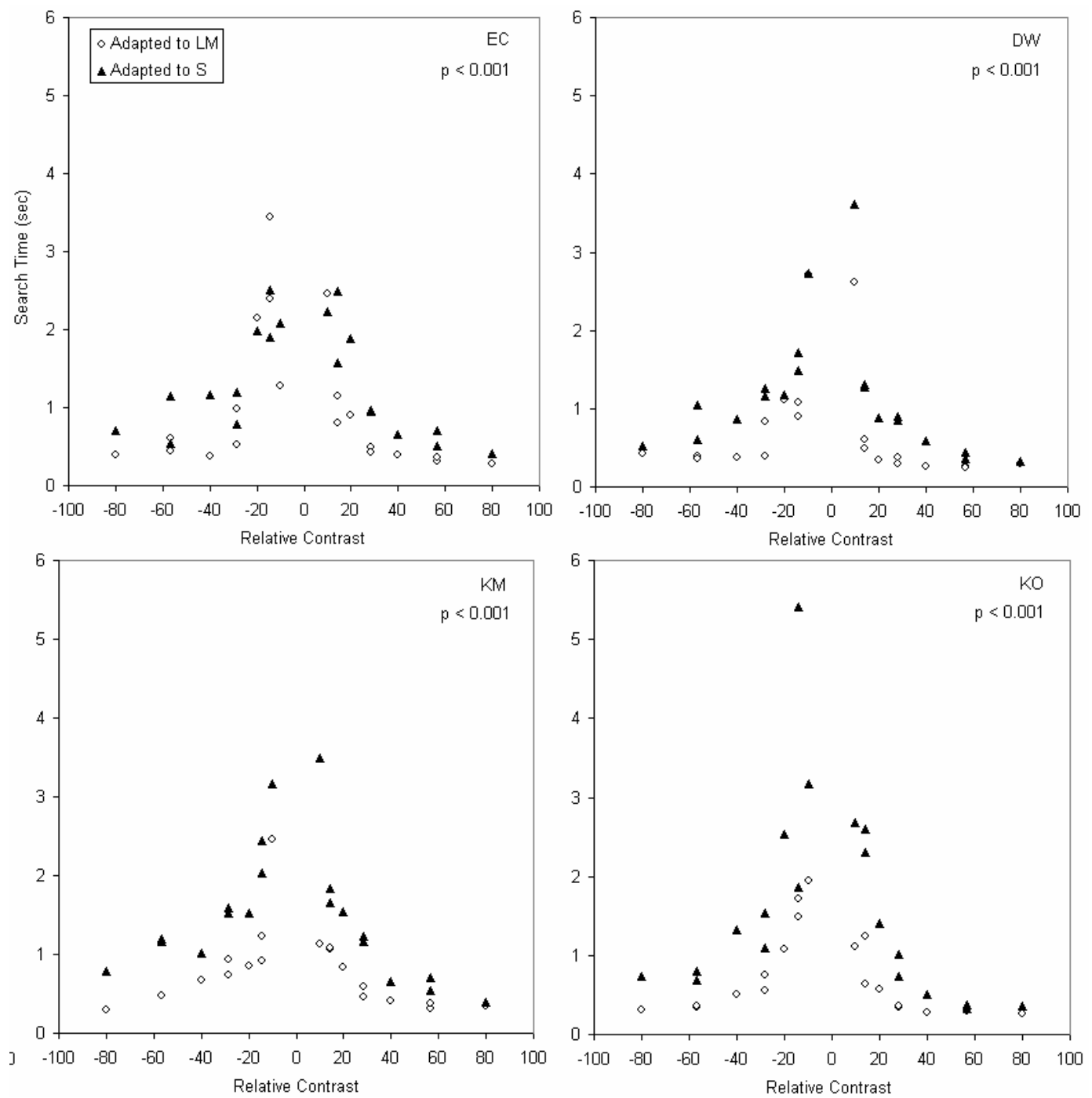

Figure 3a. Reaction times for locating targets on the LvsM background, after adapting to the LvsM (circles) or SvsLM (triangles) background. Relative contrast gives the distance of the target color from the background color axis. Each panel plots the results for a different subject. P values are from sign tests comparing search times on the adapted vs. orthogonal background, and show that search was significantly faster on the adapted background for all subjects.

within $2.5 \mathrm{deg}$ of the target center for $500 \mathrm{~ms}$. This margin was based on pilot measures of fixation accuracy for the display. Actual reported search times are from the beginning of the final fixation on the target as determined by a post hoc analysis. Saccades during the search were recorded with a criterion that eye position change by $>1$ deg during the 20 ms sampling interval. Successive saccades in the example of Figure 1 are shown by the numbered circles. Each trial was monitored by the experimenter, and a small number were omitted because the eyetracker temporarily lost the subject's eye or mislocated its position (e.g. when the subject fixated the target but the recorded position was not within $2.5 \mathrm{deg}$ ).

\section{RESULTS}

Search on the adapted vs. the orthogonal background. In the first set of conditions, we compared performance when subjects searched on the same background they had adapted to or on the orthogonal background, similar to the conditions tested by Webster et al. ${ }^{14}$. Figure 2 plots the mean reaction times for each target color as a function of its contrast or 

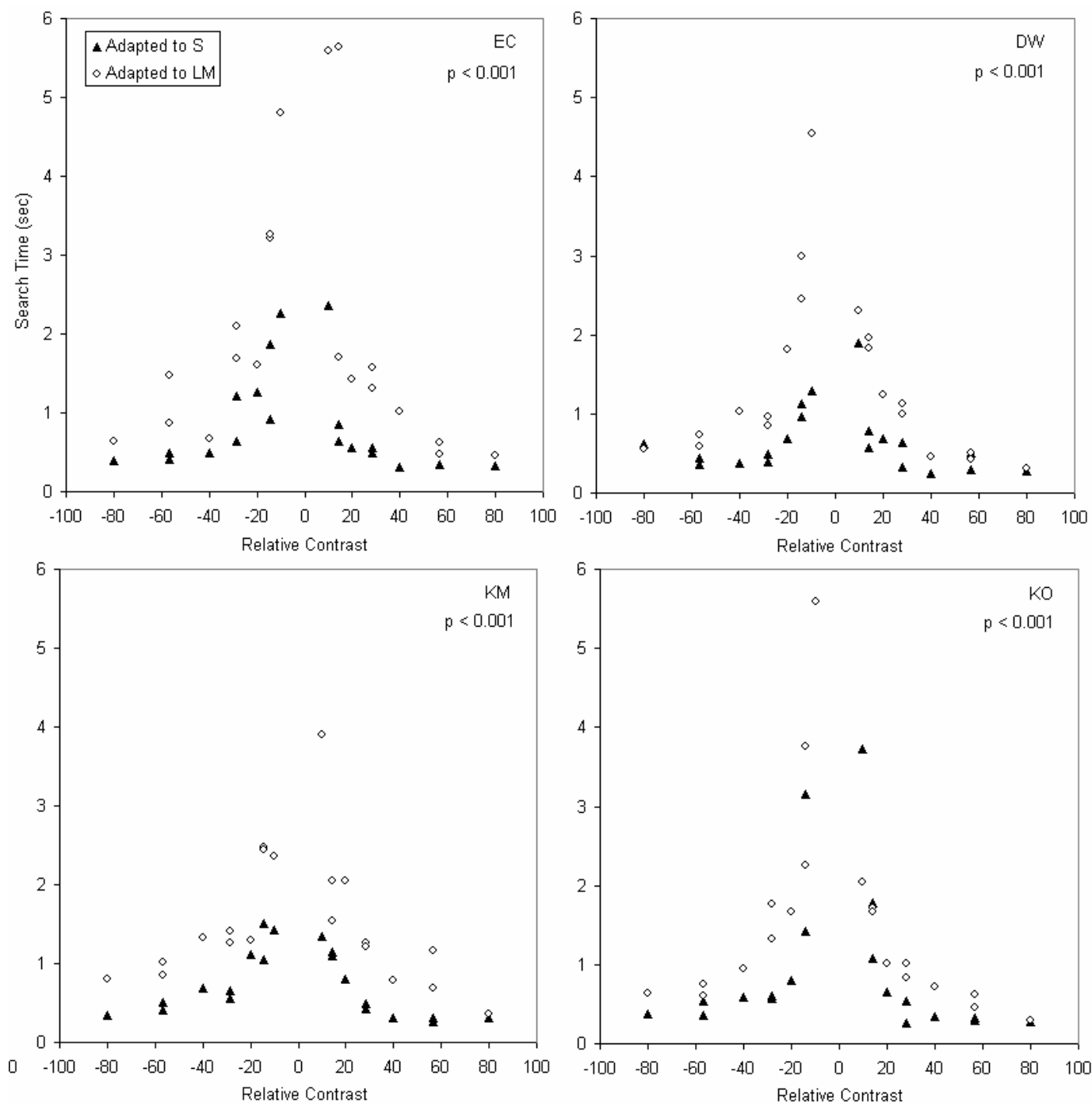

Figure 3b. Reaction times for locating targets on the SvsLM background, after adapting to the LvsM (circles) or SvsLM (triangles) background. Relative contrast gives the distance of the target color from the background color axis. Each panel plots the results for a different subject. P values are from sign tests comparing search times on the adapted vs. orthogonal background, and show that search was significantly faster on the adapted background for all subjects.

distance relative to the background axis. This is equivalent to the SvsLM component for targets shown on the LvsM background (which has an SvsLM contrast of 0), and to the LvsM component for targets shown on the SvsLM background (which has an LvsM contrast of 0 ). To more directly evaluate the effect of adaptation, the data are arranged so that each panel compares the reaction times for the same target on the same background, but after adapting either to that background or the orthogonal background. Thus the two sets of points are drawn from different adapting sessions, but similar differences are obtained when comparing reaction times on the two different test backgrounds within a single adapting session.

For each observer a substantial adaptation effect was found. That is, subjects were consistently faster at finding the targets on the LvsM background after they had first adapted to that background rather than to the SvsLM background. The differences in the mean reaction times were evaluated with a nonparametric sign test. If there were no effect of the adaptation, then on average the search times on the adapting background should be shorter only half of the time. Yet they were instead shorter nearly every time for every subject, a difference that was highly significant $(\mathrm{p}<0.001$ for all subjects 

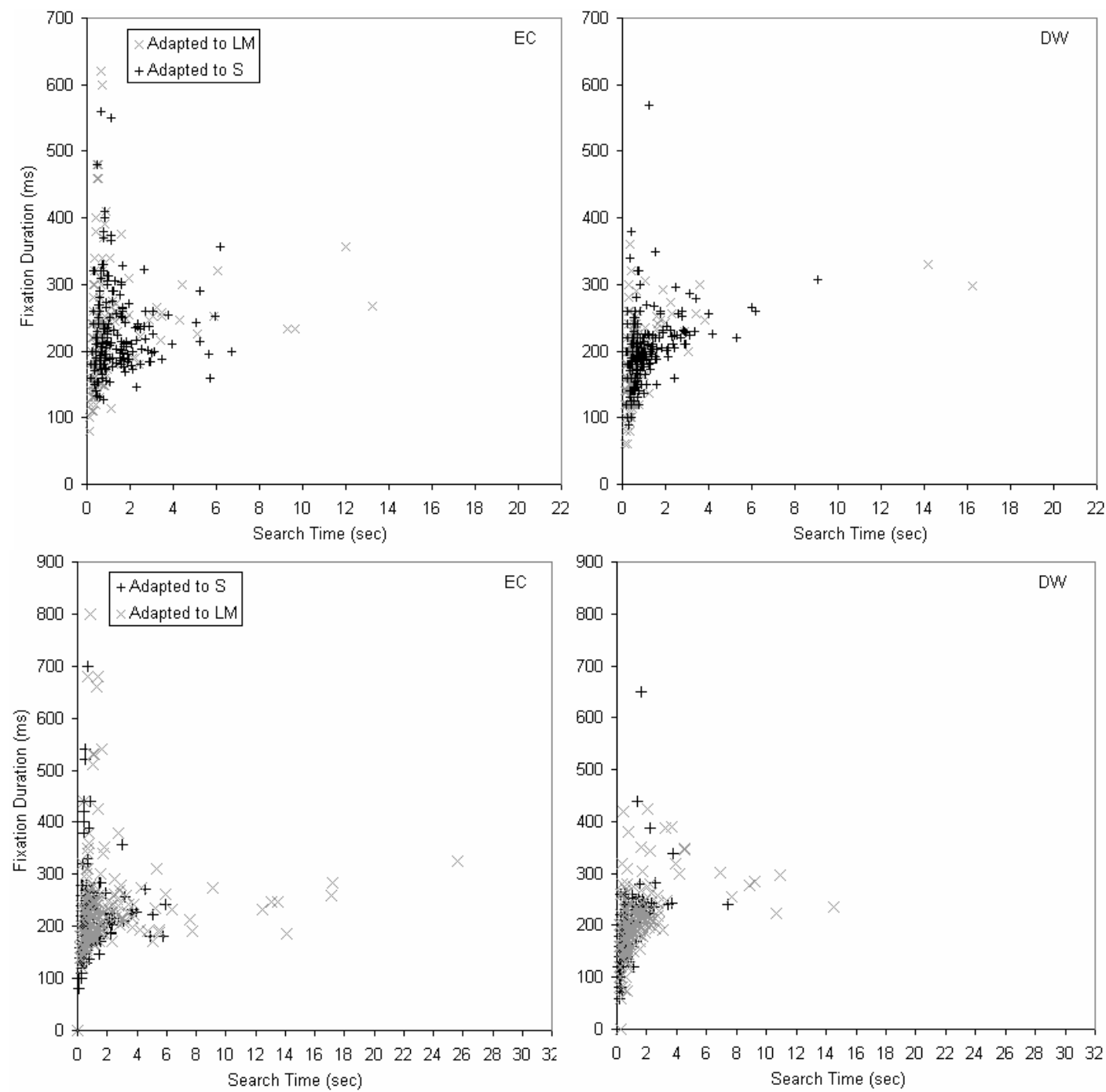

Figure 4. Mean fixation duration plotted as a function of search times for two subjects (EC and DW; results for two additional subjects were similar). Top panels: durations while searching on an LvsM background. Bottom panels: durations while searching on an SvsLM background.

and conditions). These results thus confirm the adaptation effects reported by Webster et al. ${ }^{14}$, and in fact are surprisingly stronger, since the differences they reported were less robust.

As noted, a major aim of this study was to explore the basis for this improvement. One possibility is that adaptation selectively reduced sensitivity to the adapting color axis, and thereby increased the effective difference between the target and the adapting background. By this account search times would be slower on the orthogonal background, because in that case the same adaptation would selectively reduce sensitivity to the very axis that distinguishes the target from the background. However, a second possibility is that subjects sampled the adapting and novel backgrounds in different ways. For example, if subjects can more readily encode the statistical structure of a familiar background, then they might be able to sample it with a broader or more efficient attentional spotlight. This might allow the background to be scanned with larger fixation steps and/or shorter fixation durations. Accordingly, we analyzed the pattern of the eye movements to test for differences in fixations across the adapted and unadapted backgrounds.

Figure 4 plots fixation durations for the different adapting backgrounds. Note that in these plots durations are plotted as a function of search times (rather than target contrast), in order to more directly compare scanning patterns for trials that 

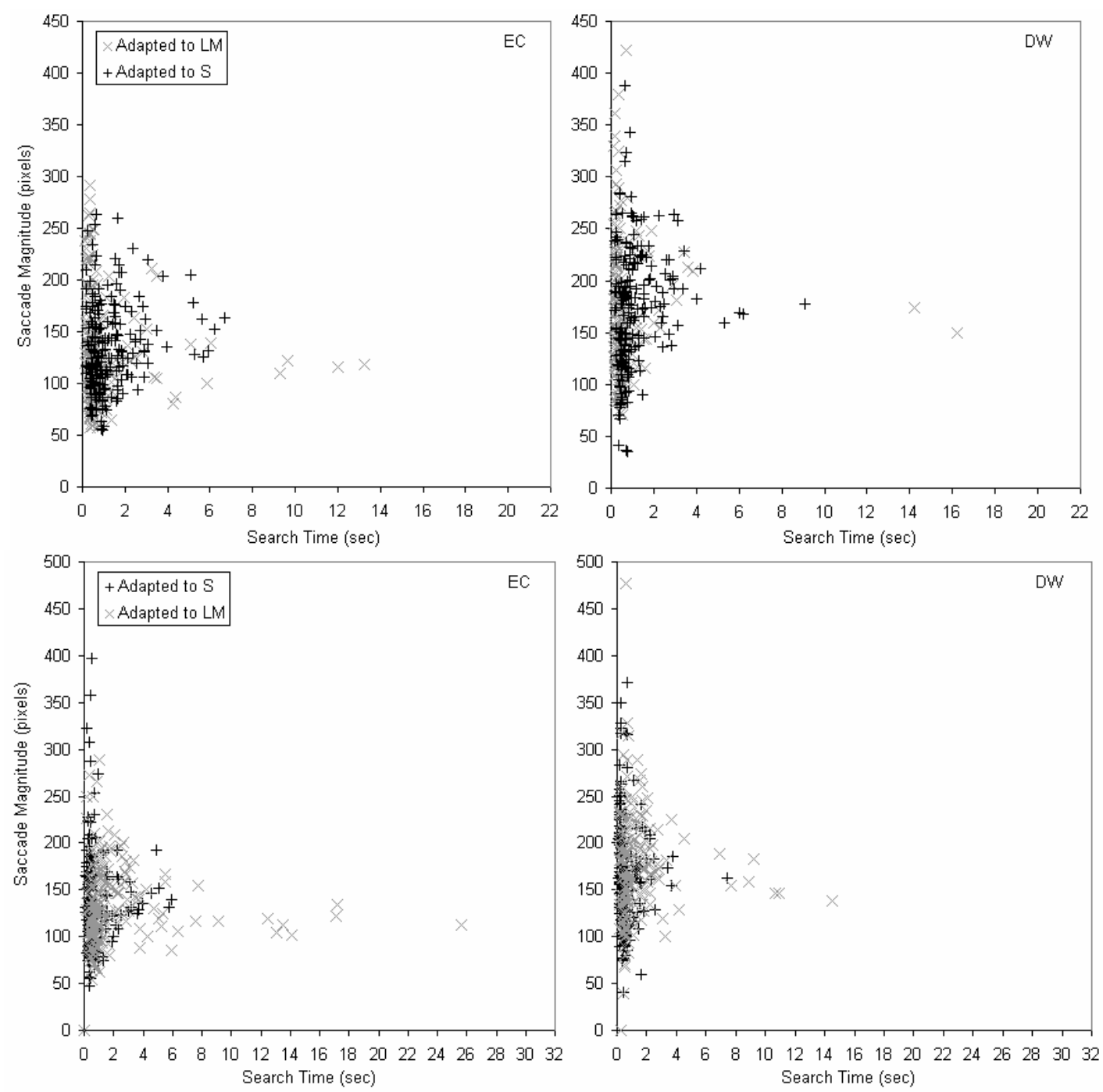

Figure 5. Mean saccade magnitude plotted as a function of search times for two subjects (EC and DW; results for two additional subjects were similar). Top panels: magnitudes while searching on an LvsM background. Bottom panels: magnitudes while searching on an SvsLM background. At the $57 \mathrm{~cm}$ viewing distance 20 pixels equaled $1 \mathrm{deg}$.

resulted in the same search times. Fixation durations showed a general increase with search times. The variance also decreased for longer search times, since the latter average over more fixations. However, these patterns were not distinguishable for the adapting and orthogonal backgrounds, suggesting that the average dwell times on either background were the same.

Figure 5 shows similar analyses of average saccade length as a function of search time. These again decrease in variance as the search time increases and more saccades are averaged, and show a weak tendency to skew toward shorter average magnitudes for longer searches. However, again the pattern of saccades appears similar for the two adapting conditions. We also explored a further analysis (not shown) in which we plotted the distribution of saccade directions. For targets that pop out the saccades should mostly point toward the target and thus the distribution of angles should be narrowly tuned around the target direction, while the range of angles should progressively increase as the target salience decreases. However, this again failed to reveal a difference between search patterns on the familiar and novel backgrounds. Of course, these simple comparisons may have missed more subtle or other potential differences in the eye movements, but based on them there is no compelling evidence for a difference in scanning strategies when observers were searching on 


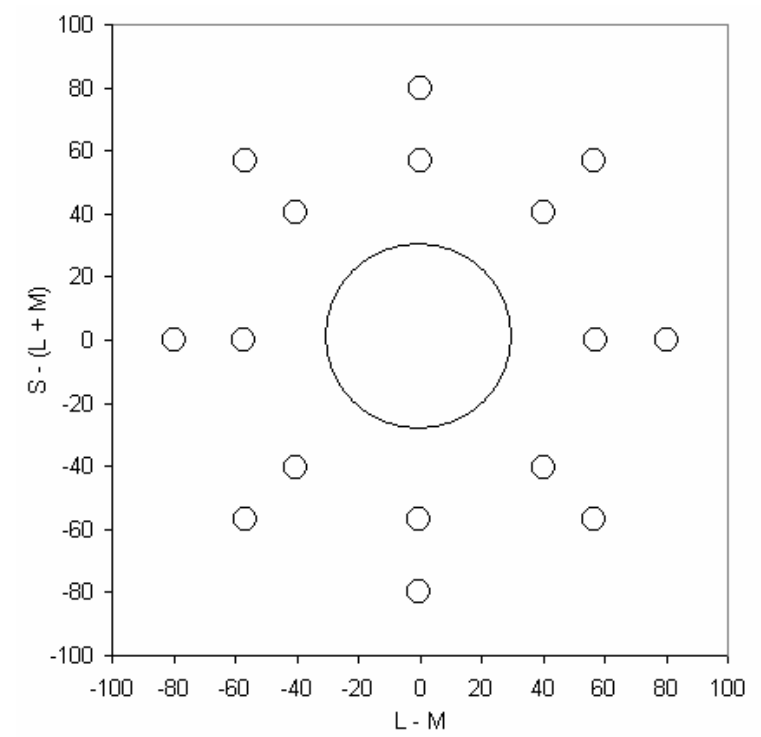

Figure 6. Color coordinates for the nonselective background (a circle of hues with a fixed chromatic contrast of 28) and the target colors, shown by smaller circles.

a background with highly familiar colors vs. suddenly novel colors. Thus this suggests that the clear differences in reaction times on the two backgrounds instead resulted from selective adaptation to the background colors. In other words, the subjects appeared to be searching in the same way, but the effective contrasts differed depending on the observer's state of adaptation.

Search on nonselective color backgrounds. Even if scan patterns were similar under the two conditions we tested, it remains possible that the improvements were not due specifically to adaptation to the chromatic properties of the background, since they might also reflect a form of learning about the colors defining the background. That is, subjects might simply have become familiar with which colors were present in the background, and thus better able to identify which targets did not belong to the background. The distinction between perceptual adaptation and perceptual learning is nebulous $^{25}$. However, the contrast changes induced by color contrast adaptation are reasonably well defined and thus should be largely predictable. In particular, adaptation to stimuli that are spatially or temporally modulated along a single axis in color space are known to induce strong and selective changes in perceived contrast along the adapting axis ${ }^{26}$. This selective change biases the perceived hue of stimuli away from the adapting axis and toward the orthogonal axis (since it reduces in any hue the component contrast along the adapting axis). Alternatively, adaptation to a nonselective color distribution (e.g. modulated along all color directions) produces a general loss in perceived contrast, and thus does not bias the hue of targets relative to the adapting distribution. In other words, improvements in performance with adaptation are only predicted to occur when the adaptation selectively reduces sensitivity to the background more than the target. Conversely, a performance change based on learning is not obviously constrained in the same way. As long as the target and background colors are distinguishable, it should be possible to learn the difference between them and take advantage of this in the search.

We therefore explored how search times were influenced by adaptation to a nonselective color distribution to try to discriminate between these alternatives. The colors defining the backgrounds and targets are shown in Figure 6. In this case observers adapted either to an achromatic background (that had the same random luminance variation in the ellipses but no variation in color) or to a background with colors drawn from a circle with a fixed radius of 28 . The background colors thus all had the same contrast but varied in hue. Target colors again spanned a range of color axes but were limited to high contrasts of 57 or 80 . In pilot runs we also included contrasts lower than the background. However, for our conditions these proved nearly impossible to find, consistent with previous studies showing that targets bounded by the background color distribution (so that they are not linearly separable from the background) are less visible ${ }^{27,28}$. 

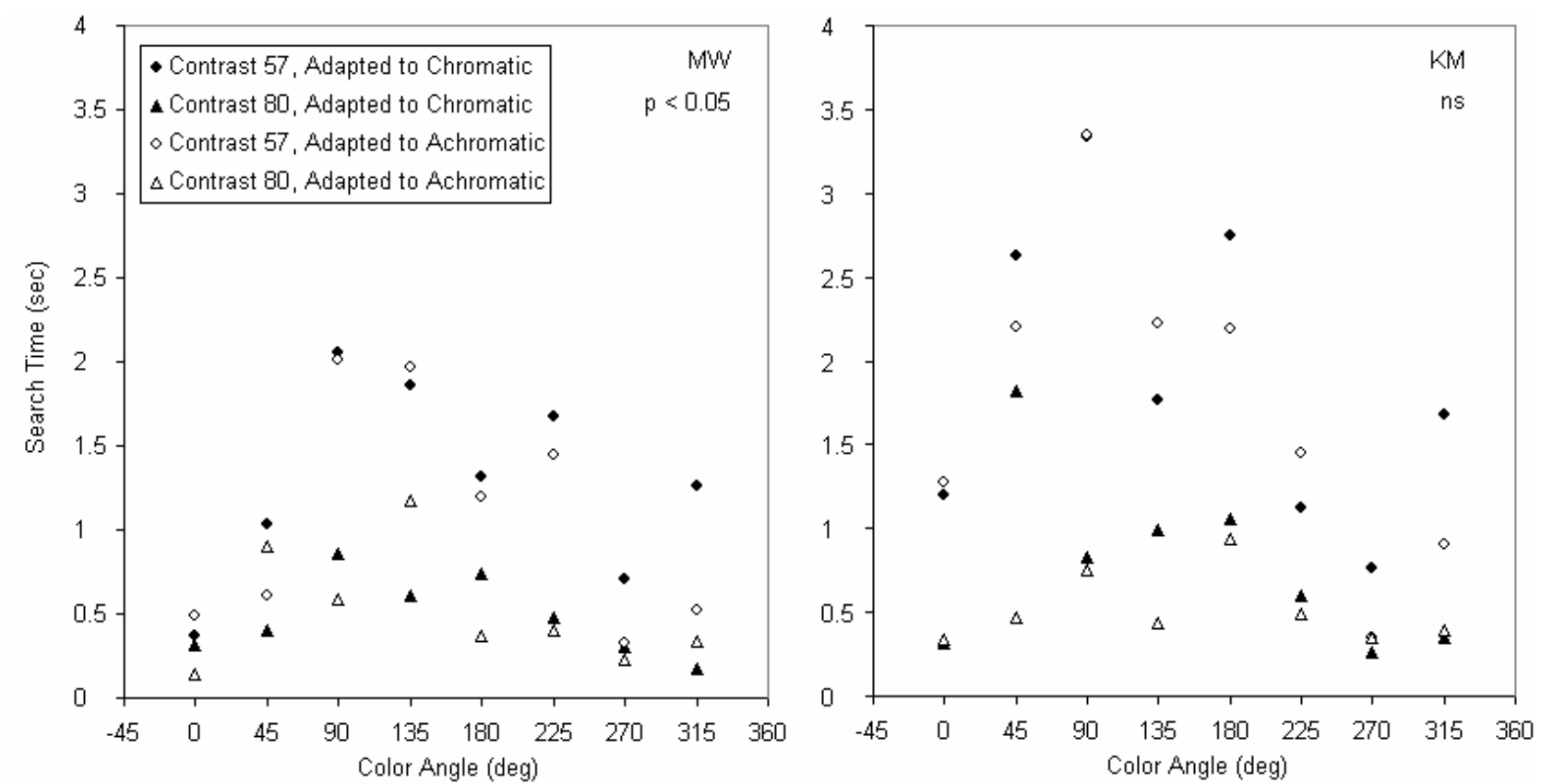

Figure 7. Search times for targets on the nonselective color background, after adapting to the color background or an achromatic background for two subjects (MW and KM; partial results for 3 further subjects were similar). Target contrasts of 57 (circles) or 80 (triangles) are plotted as a function of their angle within the LvsM and SvsLM space (see Figure 6). P values are from a sign test comparing search times for the two adapting conditions, which were significantly slower on the chromatic background for MW but did not differ for the other subjects.

Figure 7 compares the search times for each target color after adapting either to the achromatic or nonselective color background. Again, a simple interpretation of adaptation predicts no difference between these conditions while learning predicts that search should be faster on the color background. Surprisingly, if anything it was instead worse. This was again confirmed by a sign test comparing the mean reaction times across the two conditions (which reached significance for one subject but not the others). The basis for the poorer performance is unclear, though it is possible that adaptation effectively collapsed apparent contrasts along all color axes and that this reduced the apparent difference between the background and target colors. In any case, these results are inconsistent with the idea that subjects simply learned the adapting colors. Thus they again favor an account of the search performance based on adaptation induced changes in the effective contrast and therefore salience of the stimuli.

Parenthetically, it is worth noting that search times in this task also revealed a dependence on hue angle, even though the colors were selected to try to equate contrasts along the different chromatic axes. In particular, search times tended to be longer for $+\mathrm{S}$ (purple) than for $-\mathrm{S}$ (yellow-green) targets. The basis for this asymmetry is unclear, yet since it affected opposite poles of the same axis it is unlikely to reflect an artifact in how the axes were scaled.

\section{DISCUSSION}

In this study our aim was to evaluate different potential explanations for the changes in search efficiency following a period of adaptation to the stimulus context. However, the very fact that search performance improved is itself important to emphasize. While pattern-selective adaptation can profoundly alter the appearance of subsequently viewed stimuli, improvements in discrimination or detection following adaptation have been difficult to demonstrate and remain controversial $^{29}$. This may in part be because most studies testing for performance changes have focused on nearthreshold stimuli and on how adaptation influences the ability to detect changes in the adapting stimulus itself (e.g. in its contrast). We instead evaluated the effect of adaptation on the ability to detect stimuli that differed from the adapting distribution and over a range of target contrasts. The present results confirm the findings of Webster et al. ${ }^{14}$ in showing that search times are consistently faster when observers are searching on backgrounds with the same properties as the 
backgrounds they adapted to. Together these results support a functional role of adaptation in enhancing the salience of novel characteristics of the environment, as proposed by Barlow ${ }^{13}$.

The clear differences in search times for locating the targets on the adapted vs. novel backgrounds were not accompanied by measurable changes in the patterns of eye movements on the two backgrounds. Again, this suggests that the differences in performance were due to changes in the effective relative contrast of the backgrounds and targets, as predicted by a selective adaptation effect, and were not due to a change in sampling strategies. In fact, a change in fixation patterns may be implausible, for recent work suggests that fixations during a visual search task are already nearly optimal. Najemnik and Geisler ${ }^{19}$ compared scan patterns to an ideal Bayesian observer and showed that observers were close to the ideal in both search times and the statistics of the eye movements. Interestingly, the ideal observer in their analysis incorporates knowledge about the statistical structure of the scene, which in their case was $1 / \mathrm{f}$ noise characteristic of the amplitude spectra of natural images. In turn, the present results can be explained by assuming that human observers can adjust to better incorporate the varying statistics in new scenes through a process like adaptation. Note again that in our study adaptation was to consistent image statistics rather than to a specific individual scene. It remains possible that different search strategies would emerge if observers were inspecting the same scene they had adapted to.

Further evidence for an adaptation basis for the improvements in visual search comes from our second experiment, which showed that adapting to a nonselective background did not facilitate detection of the targets, and for some subjects may have impeded it. Again, a contrast adaptation effect is predicted to improve performance only in cases where the targets and backgrounds differ along the dimensions that the adaptation is selective for, for otherwise the adaptation is altering the contrast of both the target and the background in similar ways. Indeed, it is probably for this reason that measures of contrast discrimination following adaptation - i.e. detecting changes in contrast along the adapting axis have yielded only weak and inconsistent effects ${ }^{29}$.

It remains uncertain specifically how adaptation alters the salience of the targets. Adapting to the LvsM axis selectively reduces sensitivity to the LvsM contrasts in any color. Note that this does not change the distance of the target color from the LvsM axis, since this is given by the SvsLM component of the target. (If anything it may instead reduce the distance, since the LvsM adaptation also has a weak effect on contrasts along the SvsLM axis ${ }^{23}$ ). However, because the LvsM component of any off-axis target is decreased more than the SvsLM component, the perceived direction of any target will be rotated away from the adapting axis and toward the orthogonal axis, and thus adaptation will accentuate the difference in hue or chromatic angle between the target and background. For example, after adapting to the reddishgreenish colors of the LvsM axis, an orange target color should appear less red and more yellow. Webster et al. ${ }^{30}$ showed that these hue changes are large following adaptation to patterned stimuli very similar to the ones used in our search tasks. If search times were better correlated with the hue difference between the target and background than with the component contrast difference then this could provide a simple account of how adaptation alters visual salience. However, reaction times for different hue angles instead appear at least roughly comparable when equated for the distance from the background axis, and this leaves the basis for the salience changes unclear.

Humans are presumably highly efficient in visual search because the ability to detect novel or targeted information confers an obvious survival advantage. Our results show that observers become more efficient when they are allowed to adapt to the specific statistics of their current environment, and it is likely that this adaptation would similarly improve efficiency along other dimensions than the specific color environments we examined. The fact that the search performance follows the pattern predicted from established characteristics of pattern-selective adaptation suggests that models based on this adaptation could be used to predict and potentially improve performance in novel environments for either human or robotic vision. For example, our results suggest that performance in new environments could be enhanced by pre-adapting the observer to the statistics of the environment, or conversely, by pre-filtering images to adapt them to the observer.

\section{ACKNOWLEDGMENTS}

Supported by a NASA Nevada EPSCOR Collaborative Pilot Grant. 


\section{REFERENCES}

1. H. E. Pashler, The Psychology of Attention (MIT Press, Cambridge, 1998).

2. J. M. Wolfe, "Guided Search 2.0 A revised model of visual search," Psychonomic Bulletin and Review 1, 202-238 (1994).

3. S. Yantis, "Stimulus driven attentional capture," Current Directions in Psychological Science 2, 156-161 (1994).

4. H.-C. Nothdurft, "Feature analysis and the role of similarity in preattentive vision," Perception and Psychophysics 52, 355-375 (1992).

5. A. M. Treisman and G. Gelade, "A feature-integration theory of attention," Cognitive Psychology 12, 97-136 (1980).

6. R. A. Rensink, "Change detection," Annual Review of Psychology 53, 245-277 (2002).

7. R. A. Rensink, J. K. O'Regan, and J. J. Clark, "To see or not to see: The need for attention to perceive changes in scenes," Psychological Science 8, 368-373 (1997).

8. J. Triesch, D. H. Ballard, M. M. Hayhoe, and B. T. Sullivan, "What you see is what you need," Journal of Vision 3, 86-94 (2003).

9. B. Julesz, "A brief outline of the texton theory of human vision," Trends in Neuroscience 7, 41-45 (1984).

10. H. B. Barlow, "Conditions for versatile learning, Helmholtz's unconscious inference, and the task of perception," Vision Research 30, 1561-1571 (1990).

11. E. P. Simoncelli and B. A. Olshausen, "Natural image statistics and neural representation," Annual Review of Neuroscience 24, 1193-1216 (2001).

12. M. A. Webster and J. D. Mollon, "Adaptation and the color statistics of natural images," Vision Research 37, 32833298 (1997).

13. H. B. Barlow, "A theory about the functional role and synaptic mechanism of visual after-effects," in Vision: Coding and Efficiency, C. Blakemore, ed. (Cambridge University Press, Cambridge, 1990), pp. 363-375.

14. M. A. Webster, V. E. Raker, and G. Malkoc, "Visual search and natural color distributions," Human Vision and Electronic Imaging III, B. Rogowitz and T. Pappas, eds., SPIE 3299, 498-509 (1998).

15. R. H. S. Carpenter, Movements of the Eyes (Pion, London, 1988).

16. M. M. Hayhoe and D. H. Ballard, "Eye movements and natural behavior," Trends in Cognitive Science 9, 188-194 (2005).

17. B. R. Beutter, M. P. Eckstein, and L. S. Stone, "Saccadic and perceptual performance in visual search tasks. I. Contrast detection and discrimination.," Journal of the Optical Society of America A 20, 134101355 (2003).

18. J. Findlay, "Saccade target selection during visual search," Vision Research 37, 617-631 (1997).

19. J. Najemnik and W. S. Geisler, "Optimal eye movement strategies in visual search," Nature 434, 387-391 (2005).

20. G. J. Zelinsky, R. P. N. Rao, M. M. Hayhoe, and D. H. Ballard, "Eye movements reveal the spatiotemporal dynamics of visual search," Psychologcial Science 6, 448-453 (1997).

21. P. Lennie and J. A. Movshon, "Coding of color and form in the geniculostriate visual pathway (invited review)." Journal of the Optical Society of America A 22, 2013-2033 (2005).

22. J. Krauskopf, D. R. Williams, and D. W. Heeley, "Cardinal directions of color space," Vision Research 22, 11231131 (1982).

23. M. A. Webster and J. D. Mollon, "The influence of contrast adaptation on color appearance," Vision Research 34, 1993-2020 (1994).

24. D. I. A. MacLeod and R. M. Boynton, "Chromaticity diagram showing cone excitation by stimuli of equal luminance," Journal of the Optical Society America 69, 1183-1186 (1979).

25. B. A. Dosher and Z. L. Lu, "Mechanisms of perceptual learning," Vision Research 39, 3197-3221 (1999).

26. M. A. Webster, "Human colour perception and its adaptation," Network: Computation in Neural Systems 7, 587-634 (1996).

27. B. Bauer, P. Jolicoeur, and W. B. Cowan, "Visual search for colour targets that are or are not linearly separable from distractors," Vision Research 36, 1439-1465 (1996).

28. M. D'Zmura, "Color in visual search," Vision Research 31, 951-966 (1991).

29. M. A. Webster, "Pattern selective adaptation in color and form perception," in The Visual Neurosciences Volume 2, L. M. Chalupa and J. S. Werner, eds. (MIT Press, Cambridge, 2003), pp. 936-947.

30. M. A. Webster, G. Malkoc, A. C. Bilson, and M. A. Webster, "Color contrast and contextual influences on color appearance," Journal of Vision 2, 505-519 (2002). 